\title{
Single cells (put a ring on it)
}

\author{
Benjamin A. Wolfe and Michael Glotzer ${ }^{1}$ \\ Department of Molecular Genetics and Cell Biology, The University of Chicago, Chicago, Illinois 60637, USA
}

Proper spatial and temporal regulation of the small GTPase RhoA at the equatorial cortex represents a critical step in the specification of the division plane in eukaryotes. Despite increased understanding of the mechanisms whereby RhoA becomes active following chromosome segregation, far less is known about how RhoA is spatially regulated so that it concentrates precisely at the division site. In the April 1, 2009, issue of Genes \& Development, Yoshida and colleagues (pp. 810823) uncovered two genetically separable mechanisms whereby Rho1 is recruited to the bud neck in the budding yeast Saccharomyces cerevisiae to facilitate cytokinesis.

The Rho family of small GTPases (Rho, Rac, and Cdc42) regulates the actin cytoskeleton to influence biological processes as diverse as polarity establishment, migratory behavior, secretion, and cell division (Jaffe and Hall 2005). In each of these cases, Rho proteins respond to external or internal signaling cascades to locally activate downstream effectors that generate or remodel actin structures. In the canonical model for Rho family GTPase signaling, Rho family GTPases undergo conformational changes during the switch from the GDP-bound to the GTP-bound form to enable specific associations with effector proteins (Fig. 1A). Guanine nucleotide exchange factors (GEFs) promote the release of GDP for GTP and, hence, activate the GTPase. These events are reversed by GTPase-activating proteins (GAPs) that accelerate the slow intrinsic hydrolysis activity of the GTPase. Rho family proteins are also regulated by RhoGDIs, or dissociation inhibitors, that sequester the molecule away from the cortex, where it may come into proximity with GEFs. Although these different Rho GTPase regulatory factors play critical roles in modulating the levels of Rho activity, the extent to which these molecules influence the localization of Rho proteins and effector binding is far less well understood.

Localized activation and recruitment of Rho family members to distinct subcellular compartments appears to be a critical step in the generation of a robust GTPase

[Keywords: Rhol; cytokinesis; GEF; polybasic sequence; yeast] ${ }^{1}$ Corresponding author.

E-MAIL Mglotzer@uchicago.edu; FAX (773) 702-3172.

Article is online at http://www.genesdev.org/cgi/doi/10.1101/gad.1801209. signaling cascade (Inoue et al. 2005). In particular, Rho GTPases become enriched at membrane structures due to C-terminal tail lipid modifications (Seabra 1998). Under most situations, this has the effect of dramatically increasing the local concentration of the GTPase, its regulators, and its effectors. In addition to increasing the local concentrations of GTPase signaling factors, additional mechanisms are in place to either maintain or rapidly terminate GTPase signaling (Heo et al. 2006). For instance, scaffold proteins that position the GTPase in close proximity to its regulators and effectors can augment these local accumulations (Piekny and Glotzer 2008). Additionally, allosteric interactions of the GEF with either the GTPase itself or with its effectors can create an environment that sustains GTPase activation (Kitzing et al. 2007; Gureasko et al. 2008). Although not all Rho family members may use each of these mechanisms in every cell biological scenario, these studies collectively underscore the need to precisely control the spatial and temporal regulation of Rho activation and inactivation.

\section{Rho GTPases regulate cytokinesis}

Cytokinesis, the terminal event of the cell cycle that results in the production of two genetically equivalent daughter cells, represents a critical example of Rho family GTPase signaling at the cell cortex (Piekny et al. 2005). In most animal cells, the plane of cell division is specified immediately following chromosome segregation to a region on the equatorial cortex midway between the separated chromosomes. Specifically, the anaphase microtubule cytoskeleton positions the small GTPase RhoA at the equatorial cortex, where it induces assembly of an actomyosin-based contractile ring just underneath the plasma membrane at the future division site. The constriction of this contractile ring during cytokinesis pulls the overlying plasma membrane inward toward the cell center to cleave the cell in two. Because mislocalization of the contractile ring can have deleterious effects on genome stability, one of the many hallmarks of cancer, the positioning of the RhoA signal is under stringent cell cycle control both spatially and temporally. Indeed, in animal cells, redundant mechanisms collaborate to precisely define the narrow region on the cortex to which RhoA associates during anaphase (Dechant and Glotzer 2003; Bringmann and Hyman 2005; Werner et al. 2007; Chen et al. 2008; Murthy and Wadsworth 2008). Still, the 
A

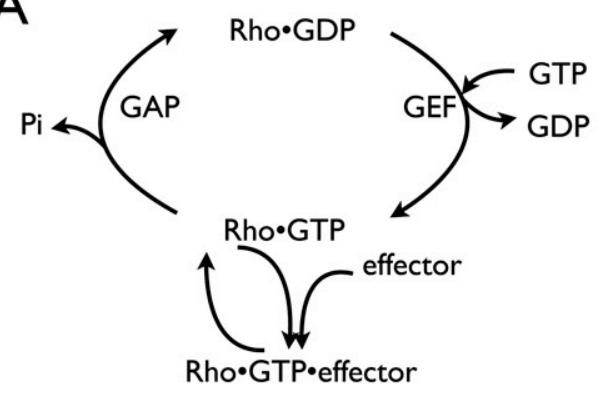

B

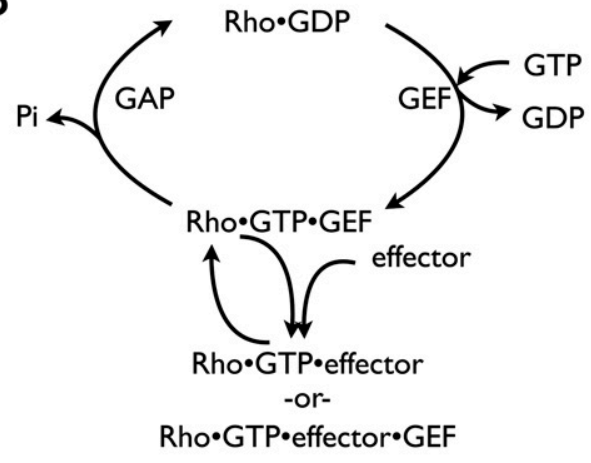

C
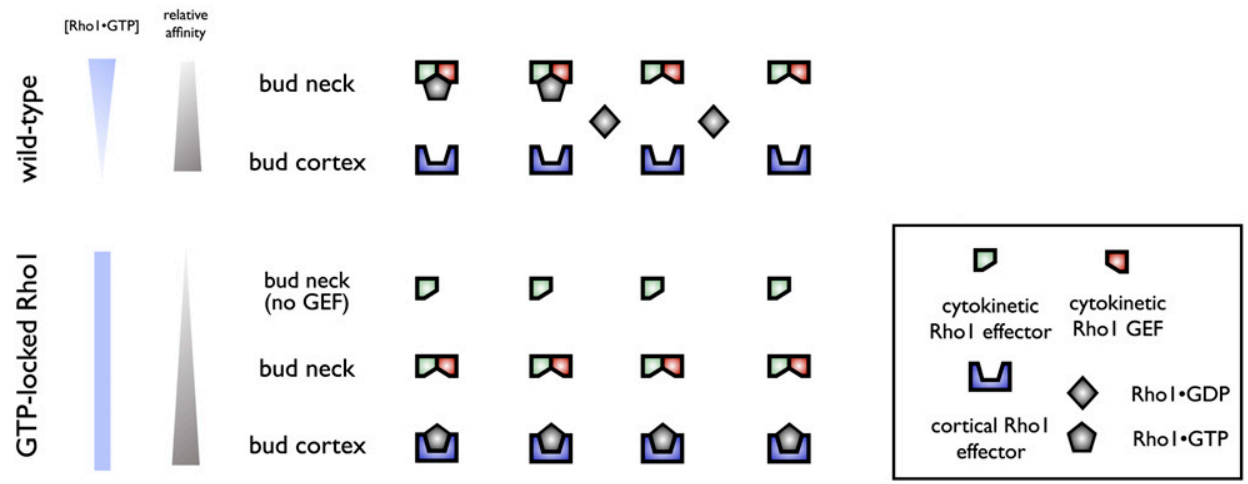

Figure 1. (A) The classical Rho GTPase cycle. Rho GTPases are activated by GEFs so that they can bind to effectors. After dissociation from effectors, GAPs can hydrolyze GTP-bound Rho to inactivate the GTPase. $(B, C)$ Models that explain why activated forms of Rho1 fail to support CAR assembly. $(B)$ The RhoGEF may be directly involved in binding of effectors to activated Rho1; they may even remain in a single protein complex. $(C)$ Alternatively, activated Rhol may fail to bind to the bud neck because it binds preferentially to ectopic sites on the bud cortex. In wild-type cells, this would not occur because Rhol is activated locally.

mechanisms responsible for local enrichment of RhoA are not completely understood.

In contrast, in other eukaryotes such as yeast and plants, the plane of division is determined prior to chromosome segregation (Balasubramanian et al. 2004). In such situations, nuclear division must be coordinated with respect to the predetermined plane of division to ensure that half the genetic material and cytoplasmic contents are inherited by each of the daughter cells following division. The budding yeast Saccharomyces cerevisiae represents an excellent model system for the study of cell division. In this organism, division is accomplished through construction and growth of a daughter bud and the alignment and segregation of the genetic material and cytoplasmic contents into the bud, followed by cell cleavage at the mother-bud neck. Similar to the situation in higher eukaryotes, a contractile ring (CAR, cytokinetic actin ring/_containing actin, myosin, and associated proteins-forms at the bud neck following chromosome segregation and contributes to the ingression of the membrane material as well as the deposition of septal material to facilitate cytokinesis. Whereas the actomyosin ring is absolutely essential for cell division in most eukaryotes, such a structure is not absolutely required for cytokinesis in budding yeast (Vallen et al. 2000). This is because budding yeast possess an alterna- tive pathway (CAR-independent) capable of dividing the cell into equal parts that relies on the construction of a secondary septum. While either of these pathways is sufficient for viability, they are likely to be coordinated in some manner.

Consistent with its broad-based requirement for assembly of the CAR in eukaryotes, budding yeast Rhol (homologous to mammalian RhoA) becomes concentrated at the bud neck during late mitosis and is indispensable for CAR assembly and cytokinesis (Tolliday et al. 2002; Yoshida et al. 2006, 2009). Analogous to the mechanism in higher eukaryotes (Takaki et al. 2008), Rhol concentration at the bud neck requires the activity of the Polo-like kinase in budding yeast, Cdc5 (Yoshida et al. 2006). Cdc5 is part of the mitotic exit network (MEN), a pathway that couples the inactivation of mitotic Clb-Cdk activity with the initiation of cytokinesis during the exit from mitosis (Jaspersen et al. 1998; Visintin et al. 1998; Yoshida et al. 2002). While other MEN mutants properly assemble CARs that then fail to constrict due to the absence of mitotic exit (Yoshida et al. 2006), mutants in Cdc5 fail to recruit critical CAR components to the bud neck in anaphase, suggesting a more direct role for Cdc5 in CAR assembly. Consistent with this, temperaturesensitive mutants of Cdc5 (cdc5-2) possess decreased amounts of Rhol-GTP (Yoshida et al. 2006), suggesting 
that Cdc5 controls global Rho1 activity. In addition to this control of global Rho1 activation, Cdc5 specifically targets the RhoGEFs Tus1 and Rom2 for phosphorylation and for subsequent recruitment to the bud neck during anaphase, where they can locally activate Rhol to promote CAR assembly. RhoGEF mutants incapable of associating with Cdc5 generate an activated form of Rhol that fails to accumulate at the bud neck, thus separating Rhol activation from its proper bud neck localization. Conversely, this same group has shown that the requirement for Cdc5 in CAR assembly could be partially bypassed by fusing the RhoGEF Tus1 to a resident bud neck protein (Yoshida et al. 2006). Hence, the Polo kinasedependent positioning of RhoGEFs at the division site appears to be a critical step in Rhol recruitment and CAR assembly. Despite this importance, the mechanism(s) by which RhoGEF phosphorylation triggers Rhol bud neck accumulation have not been determined, nor is it understood whether the localized activation of Rhol is the sole function of RhoGEFs in promoting CAR assembly.

\section{RhoGEF-mediated Rho recruitment}

In the April 1, 2009, issue of Genes \& Development, Yoshida et al. (2009) addressed some of these open questions by identifying the mechanism(s) responsible for concentrating Rhol at the division plane during mitotic exit. In doing so, the investigators uncovered two distinct modes by which Rhol accumulates at the bud neck. The first, occurring during late anaphase as the CAR first assembles, is RhoGEF-dependent and is proposed to rely on cycling of the GTPase. The second mechanism involves the association of the Rhol polybasic sequence with a phosphoinositide membrane environment that forms at the bud neck following the exit from mitosis. These divergent mechanisms for Rhol recruitment also contribute to distinct cytokinesis pathways: The former is necessary for CAR-dependent cytokinesis, while the latter contributes to CAR-independent cytokinesis. These data underscore the ubiquitous role that Rho GTPases play in essential cellular processes, such as cytokinesis, and highlight the disparate and redundant mechanisms that ensure Rho GTPases fulfill their necessary functions.

To more precisely define a mechanism by which Polo kinase promotes Rhol bud neck recruitment, Yoshida et al. (2009) determined whether the defective CAR assembly in cdc5-2 cells could be rescued by expression of a GTP-trapped mutant of Rhol (Rho1-Q68H). Although Rho1-Q68H can fulfill Rhol function in many other cellular processes, it is incapable of supporting CAR assembly in the absence of Cdc5 function, suggesting that Cdc5 plays additional roles in CAR assembly other than the generation of Rhol-GTP. Although GTP-trapped Rhol does not interfere with CAR assembly in the presence of endogenous Rho1, Rho1-Q68H behaves as a loss-of-function mutant with respect to localization to the bud neck and for CAR assembly when endogenous Rhol activity is compromised, thus providing a molecular explanation for its failure to rescue cdc5-2 cells. In con- trast, another activated Rhol mutant behaves in a slightly different manner: A Rhol mutant that is predicted to rapidly dissociate bound nucleotides can localize to the bud neck. Because RhoGEFs associate with either the GDP-bound or nucleotide-free forms of GTPases, the investigators hypothesized that recruitment of Rhol to the plane of division in budding yeast requires the activity of RhoGEFs. In good accordance with this model, a nucleotide-free Rhol mutant, which is predicted to associate with RhoGEFs, also localizes to the bud neck. Furthermore, using the awesome power of budding yeast genetics, the investigators made use of a yeast strain lacking several RhoGEFs (Rom1, Rom2, and Tus1) to ask whether RhoGEF function is required to concentrate Rhol to the bud neck during anaphase. Although deletion of RhoGEFs results in inviability, coordinately deleting one of two specific RhoGAPs allows strain viability. This enabled the investigators to unambiguously demonstrate that Rhol localization during anaphase requires the RhoGEFs that mediate CAR assembly, and they identify Tus1 as the most critical of the RhoGEFs for this process. Surprisingly, although anaphase recruitment of Rhol was blocked in these cells, it did accumulate at the bud neck at the time of spindle disassembly (see below).

Some functions of Rhol, even its essential functions, can be supported by a constitutively active form, presumably because its relevant effectors are prelocalized or their localization is not critical. In stark contrast, Rhol's role in CAR assembly requires RhoGEF activity. This difference may be due to an absence of prelocalized effectors of contractile ring assembly, or perhaps they may only bind to Rhol in the context of the RhoGEF. Additional protein interaction domains in the RhoGEF could provide additional binding energy for the Rholeffector complex (Fig. 1B). Finally, the failure of Rho1$\mathrm{Q} 68 \mathrm{H}$ to support CAR assembly may be due to its interaction with other, inappropriate, targets. These targets may outcompete for CAR function by virtue of higher affinity or higher abundance (Fig. 1C). Consistent with this view, during times at which Rhol would normally be localized to the bud neck, the GTP-locked mutant appears to concentrate on the cortex of the bud. As a means to test these models, a bud neck-localized form of activated Rhol could be assayed for CAR assembly. Likewise, deletion of the targets on the bud cortex might reduce the competition and allow activated Rhol to localize to the bud neck. Both of these models beg the question as to the identity of the bud neck anchor. Does the bud neck contain any specific docking sites for Rhol or its effectors other than RhoGEFs? The available evidence is consistent with a model in which the RhoGEF is upstream of Rhol localization, which in turn recruits Rhol effectors. If correct, activated forms of Rhol would not have any means of localizing.

Although the data indicate that Rhol accumulation at the bud neck is mediated by binding to its exchange factor, this association itself is not sufficient for CAR assembly, which requires interactions with effectors. To a first approximation, cycling-competent Rhol and nucleotide-free derivatives localize similarly to the bud 
neck. Cycling-competent Rhol presumably binds GTP and subsequently dissociates from the active site of the RhoGEF. Because the protein remains localized, other mechanisms must ensure that active Rhol is retained at the bud neck. Presumably, a combination of Rhol effectors and/or scaffold proteins mediate this accumulation. The RhoGEF itself may contribute to Rhol retention through its citron homology domain that is predicted to have Rho-binding activity. As Cdc5 activity is required to maintain a properly assembled CAR, a constant flux of Rho1 signaling probably occurs at the bud neck. Thus, although wild-type and nucleotide-free Rho accumulate similarly to the bud neck, the dynamics of the protein may differ in these two cases. Further, it is possible that specific factors that are important for localization of the wild-type protein are dispensable for localization of the nucleotide-free Rhol derivatives. These questions can now be addressed.

\section{A second pathway for Rho1 accumulation}

Once Rhol accumulates at the bud neck, it remains concentrated for $\sim 10 \mathrm{~min}$, during which time cell division completes. Although activated forms of Rhol fail to accumulate at the bud neck during the early stages of mitotic exit, they can accumulate during the late stages of this process. Additionally, in contrast to the dependence of the early anaphase localization of Rhol to the bud neck on the pertinent RhoGEFs, this later window of accumulation was found to be independent of the same RhoGEFs. Together, these data suggest that genetically separable mechanisms recruit Rhol to the division site. The C-terminal tail of Rhol contains a CaaX box at its C terminus that undergoes a series of modifications directing the GTPase to the plasma membrane, including the addition of a prenyl group on the cysteine residue (Seabra 1998). A fusion between GFP and the C-terminal tail of Rhol, encompassing both its CaaX box and a set of basic residues $\mathrm{N}$-terminal to the $\mathrm{CaaX}$ box, was sufficient to direct accumulation of GFP to the bud neck following mitotic exit. This late recruitment coincides with the splitting of the single ring of septin filaments at the bud neck into two distinct rings, an event that creates a diffusional barrier at the bud neck facilitating the concentration of factors important for cytokinesis (Dobbelaere and Barral 2004). This recruitment appears to be mediated by an electrostatic interaction between the C-terminal basic tail of Rhol and specific acidic phospholipids. Yoshida et al. (2009) speculate that septin rings may create a specific domain of plasma membrane particularly enriched in phosphoinositides, such as PIP2, that recruits Rhol through association with its tail domain. This second, functionally distinct recruitment of Rhol contributes to CAR-independent cytokinesis, likely by promoting local deposition of secondary cell wall materials, as Rhol is required for bud neck recruitment of a chitin synthase and likely other effectors of this pathway. Because the recruitment of Rhol during this phase of cytokinesis does not require its exchange factor, a constitutively active form of Rhol can provide this function.

\section{Comparisons with animal cells}

It remains to be seen to what extent these mechanisms may operate in organisms that do not have the same requirements and redundancies for cell division. Indeed, even in budding yeast, Rhol uses two distinct mechanisms to become localized to nearly the same cellular position during late mitosis to facilitate two disparate, but redundant, pathways regulating cytokinesis. Still, many parallels can be drawn in higher eukaryotes, underscoring the conservation of mechanisms regulating Rho GTPase function in processes such as cytokinesis.

In some large embryos, RhoA accumulates dramatically to the equatorial cortex (Mabuchi et al. 1993). Further, in Xenopus and sea urchins, a probe that binds to active RhoA accumulates at the equatorial cortex prior to furrow ingression (Bement et al. 2006). However, in human cells, it has been difficult to detect a pool of active RhoA at the equatorial cortex prior to furrowing onset. A series of complementary approaches have been used to examine whether active RhoA accumulates at the equatorial cortex following chromosome segregation. Given that active RhoA is genetically required for ring assembly, it is likely that RhoA acts locally to perform this function at the earliest stages of furrow formation. Although a FRET-based reporter does not reveal an early pool of active RhoA before furrow ingression (Yoshizaki et al. 2003), a fluorescently tagged heterologous Rho protein and an unusual fixation method both indicate that RhoA becomes active at the equatorial cortex following anaphase onset, just prior to the onset of furrowing (Yonemura et al. 2004; Yuce et al. 2005). Specifically, a GFP fusion with RhoA from nematodes localizes to the equatorial cortex prior to furrowing when expressed in human cells. Despite this localization, a similar fusion between GFP and human RhoA does not visibly accumulate at the cell equator (Yuce et al. 2005). As sequence divergence is largely confined to the extreme $\mathrm{C}$ terminus of these proteins, the additonal lysine residues present in nematode RhoA may facilitate similar electrostatic interactions with acidic phospholipids present at the equatorial cortex in human cells. Indeed, systematic analysis has shown that many small GTPases are targeted to plasma membranes via an interaction between a C-terminal basic region and phosphorylated forms of phosphatidylinositides (Heo et al. 2006). Indicative of conservation, these phospholipids also accumulate at the division site in a number of human cell lines as well as in Dictyostelium cells (Field et al. 2005; Janetopoulos and Devreotes 2006).

As in budding yeast, RhoGEFs play prominent roles in RhoA recruitment to the division site in higher eukaryotes. Ect2 is a key RhoGEF necessary for the cortical accumulation of RhoA during cell division. Ect2 is recruited to the central spindle microtubules in a cell cycledependent manner through its association with the RhoGAP component of the centralspindlin complex, 
MgcRacGAP/Cyk-4 (hereafter referred to as Cyk-4). In addition to the $\mathrm{Dbl}$ homology domain and the adjacent plekstrin homology domain, Ect2 possesses tandem BRCA1 C-terminal repeats (BRCT) in the $\mathrm{N}$ terminus that mediate its association with Cyk-4. The major Pololike kinase in human cells, Plk1, phosphorylates Cyk-4 and creates a phosphoepitope recognized by the BRCT domains of Ect2 (Wolfe et al. 2009). Thus, the recruitment of the RhoGEF Ect2 to the division site depends on the activity of a Polo kinase. In addition to its localization to the central spindle microtubules, the PH domain of Ect2 promotes cortical localization of the RhoGEF (Chalamalasetty et al. 2006), which may facilitate its association with RhoA and its effectors. Hence, properly positioning Ect2 at the central spindle to a region just underlying the equatorial cortex enhances RhoA localization and the generation of an actomyosin-based contractile ring. In addition to the central spindle pathway, the local inhibition of RhoA accumulation by polar microtubules functions as a redundant mechanism to ensure that RhoA concentrates at the equatorial cortex. In this context, a delocalized form of Ect2 suffices to facilitate RhoA localization in the absence of the central spindle pathway. This sufficiency experiment does not, however, rule out the possibility that the RhoGEF plays a more direct role in RhoA stabilization at the equatorial cortex under normal cellular conditions.

Ect2 not only interacts with RhoA, it also associates with, and appears activated by, the Rho family GAP Cyk-4, which in turn binds to anillin (Yuce et al. 2005; Zhao and Fang 2005; Kamijo et al. 2006; Nishimura and Yonemura 2006; D'Avino et al. 2008; Gregory et al. 2008). Each of these proteins has a domain that can bind to RhoA (Piekny and Glotzer 2008). Because anillin localizes to the equatorial cortex and also possesses actin- and mysoin-binding domains, it may function as a scaffold on which these GTPase regulators and effectors concentrate at the division plane. These associations likely recruit specific RhoA effectors and raise the possibility that a single molecule of RhoA could remain in the same complex, yet demonstrate a plethora of interactions. Thus, Ect2 may facilitate interactions of RhoA with effectors, as suggested by the data of Yoshida et al. (2009). Interestingly, in both budding yeast and Xenopus embryos, global expression of activated forms of RhoA do not dominantly prevent contractile ring assembly at the expected position (Drechsel et al. 1997). In light of the data from Yoshida et al. (2009), a parsimonious explanation is that because the activated protein does not associate with the localized RhoGEF, as the wild-type protein does, it may not compete efficiently for limiting cytokinetic effectors.

The requirement for RhoGEF activity to localize Rhol to the bud neck suggests that cycles of activation and inactivation are necessary for proper Rhol signaling. This is reminiscent of the GTPase flux model that has been put forth in higher eukaryotes (Miller and Bement 2009). In Xenopus embryos, inactivation of the GAP domain by a point mutation in Cyk-4 produces a less-focused zone of active RhoA signaling, suggesting that in addition to continual RhoGEF activation at the division plane, inactivation of RhoA signaling also continually refines and focuses the signal. Deletion of the entire GAP domain from Xenopus Cyk-4 likewise expands the active RhoA zone; it additionally fails to maintain active RhoA at the equatorial cortex as it moves back and forth laterally in the plane of division (Miller and Bement 2009). Because Cyk-4 $\triangle$ GAP lacks the ability to associate with RhoA, these data suggest that under normal conditions Cyk-4 facilitates anchoring of RhoA to the equatorial cortex. Thus, it seems likely that RhoGAPs will also play roles in RhoA retention as well and, depending on their regulation, they may contribute to the overall maintenance of RhoA-GTP at the cell equator.

Although the data for animal cells is not as complete as in yeast, there are indications that both phospholipid binding and interactions with a RhoGEF may contribute to localize RhoA to the division plane. Unlike yeast cells, where the cleavage plane is predetermined, in animal cells the entire cortex is responsive to the spindle, and a cleavage furrow can be induced to form at any site. Thus, there cannot be any prelocalized anchor for RhoA. In fact, relocalization of the spindle causes the zone of RhoA accumulation to adjust accordingly, ultimately repositioning the furrow (Bement et al. 2005). It is evident that even when cytokinesis in different organisms appears divergent, they are often mediated by common underlying mechanisms.

\section{References}

Balasubramanian, M.K., Bi, E., and Glotzer, M. 2004. Comparative analysis of cytokinesis in budding yeast, fission yeast and animal cells. Curr. Biol. 14: R806-R818. doi: 10.1016/ j.cub.2004.09.022.

Bement, W.M., Benink, H.A., and von Dassow, G. 2005. A microtubule-dependent zone of active RhoA during cleavage plane specification. J. Cell Biol. 170: 91-101.

Bement, W.M., Miller, A.L., and von Dassow, G. 2006. Rho GTPase activity zones and transient contractile arrays. Bioessays 28: 983-993.

Bringmann, H. and Hyman, A.A. 2005. A cytokinesis furrow is positioned by two consecutive signals. Nature 436: 731-734.

Chalamalasetty, R.B., Hummer, S., Nigg, E.A., and Sillje, H.H. 2006. Influence of human Ect2 depletion and overexpression on cleavage furrow formation and abscission. J. Cell Sci. 119: 3008-3019.

Chen, W., Foss, M., Tseng, K.F., and Zhang, D. 2008. Redundant mechanisms recruit actin into the contractile ring in silkworm spermatocytes. PLOS Biol. 6: e209. doi: 10.1371/ journal.pbio.0060209.

D'Avino, P.P., Takeda, T., Capalbo, L., Zhang, W., Lilley, K.S., Laue, E.D., and Glover, D.M. 2008. Interaction between Anillin and RacGAP50C connects the actomyosin contractile ring with spindle microtubules at the cell division site. J. Cell Sci. 121: 1151-1158.

Dechant, R. and Glotzer, M. 2003. Centrosome separation and central spindle assembly act in redundant pathways that regulate microtubule density and trigger cleavage furrow formation. Dev. Cell 4: 333-344.

Dobbelaere, J. and Barral, Y. 2004. Spatial coordination of cytokinetic events by compartmentalization of the cell cortex. Science 305: 393-396. 
Drechsel, D.N., Hyman, A.A., Hall, A., and Glotzer, M. 1997. A requirement for Rho and $\mathrm{Cdc} 42$ during cytokinesis in Xenopus embryos. Curr. Biol. 7: 12-23.

Field, S.J., Madson, N., Kerr, M.L., Galbraith, K.A., Kennedy, C.E., Tahiliani, M., Wilkins, A., and Cantley, L.C. 2005. PtdIns(4,5)P2 functions at the cleavage furrow during cytokinesis. Curr. Biol. 15: 1407-1412.

Gregory, S.L., Ebrahimi, S., Milverton, J., Jones, W.M., Bejsovec, A., and Saint, R. 2008. Cell division requires a direct link between microtubule-bound RacGAP and anillin in the contractile ring. Curr. Biol. 18: 25-29.

Gureasko, J., Galush, W.J., Boykevisch, S., Sondermann, H., BarSagi, D., Groves, J.T., and Kuriyan, J. 2008. Membranedependent signal integration by the Ras activator Son of sevenless. Nat. Struct. Mol. Biol. 15: 452-461.

Heo, W.D., Inoue, T., Park, W.S., Kim, M.L., Park, B.O., Wandless, T.J., and Meyer, T. 2006. PI(3,4,5)P3 and PI(4,5)P2 lipids target proteins with polybasic clusters to the plasma membrane. Science 314: 1458-1461.

Inoue, T., Heo, W.D., Grimley, J.S., Wandless, T.J., and Meyer, T. 2005. An inducible translocation strategy to rapidly activate and inhibit small GTPase signaling pathways. Nat. Methods 2: $415-418$.

Jaffe, A.B. and Hall, A. 2005. Rho GTPases: Biochemistry and biology. Annu. Rev. Cell Dev. Biol. 21: 247-269.

Janetopoulos, C. and Devreotes, P. 2006. Phosphoinositide signaling plays a key role in cytokinesis. J. Cell Biol. 174: 485-490.

Jaspersen, S.L., Charles, J.F., Tinker-Kulberg, R.L., and Morgan, D.O. 1998. A late mitotic regulatory network controlling cyclin destruction in Saccharomyces cerevisiae. Mol. Biol. Cell 9: 2803-2817.

Kamijo, K., Ohara, N., Abe, M., Uchimura, T., Hosoya, H., Lee, J.S., and Miki, T. 2006. Dissecting the role of Rho-mediated signaling in contractile ring formation. Mol. Biol. Cell 17: 43-55.

Kitzing, T.M., Sahadevan, A.S., Brandt, D.T., Knieling, H., Hannemann, S., Fackler, O.T., Grosshans, J., and Grosse, R. 2007. Positive feedback between Dial, LARG, and RhoA regulates cell morphology and invasion. Genes \& Dev. 21: $1478-1483$.

Mabuchi, I., Hamaguchi, Y., Fujimoto, H., Morii, N., Mishima, M., and Narumiya, S. 1993. A rho-like protein is involved in the organisation of the contractile ring in dividing sand dollar eggs. Zygote 1: 325-331.

Miller, A.L. and Bement, W.M. 2009. Regulation of cytokinesis by Rho GTPase flux. Nat. Cell Biol. 11: 71-77.

Murthy, K. and Wadsworth, P. 2008. Dual role for microtubules in regulating cortical contractility during cytokinesis. J. Cell Sci. 121: 2350-2359.

Nishimura, Y. and Yonemura, S. 2006. Centralspindlin regulates ECT2 and RhoA accumulation at the equatorial cortex during cytokinesis. J. Cell Sci. 119: 104-114.

Piekny, A.J. and Glotzer, M. 2008. Anillin is a scaffold protein that links RhoA, actin, and myosin during cytokinesis. Curr. Biol. 18: 30-36.

Piekny, A., Werner, M., and Glotzer, M. 2005. Cytokinesis: Welcome to the Rho zone. Trends Cell Biol. 15: 651-658.

Seabra, M.C. 1998. Membrane association and targeting of prenylated Ras-like GTPases. Cell. Signal. 10: 167-172.

Takaki, T., Trenz, K., Costanzo, V., and Petronczki, M. 2008. Polo-like kinase 1 reaches beyond mitosis-cytokinesis, DNA damage response, and development. Curr. Opin. Cell Biol. 20: $650-660$.

Tolliday, N., VerPlank, L., and Li, R. 2002. Rho1 directs forminmediated actin ring assembly during budding yeast cytokinesis. Curr. Biol. 12: 1864-1870.
Vallen, E.A., Caviston, J., and Bi, E. 2000. Roles of Hoflp, Bnilp, Bnrlp, and myolp in cytokinesis in Saccharomyces cerevisiae. Mol. Biol. Cell 11: 593-611.

Visintin, R., Craig, K., Hwang, E.S., Prinz, S., Tyers, M., and Amon, A. 1998. The phosphatase Cdc14 triggers mitotic exit by reversal of Cdk-dependent phosphorylation. Mol. Cell 2: 709-718.

Werner, M., Munro, E., and Glotzer, M. 2007. Astral signals spatially bias cortical myosin recruitment to break symmetry and promote cytokinesis. Curr. Biol. 17: 1286-1297.

Wolfe, B., Takaki, T., Petronczki, M., and Glotzer, M. 2009. Pololike kinase 1 directs assembly of the HsCyk-4 RhoGAP/Ect2 RhoGEF complex to initiate cleavage furrow formation. PLOS Biology (in press).

Yonemura, S., Hirao-Minakuchi, K., and Nishimura, Y. 2004. Rho localization in cells and tissues. Exp. Cell Res. 295: 300-314.

Yoshida, S., Asakawa, K., and Toh-e, A. 2002. Mitotic exit network controls the localization of Cdc14 to the spindle pole body in Saccharomyces cerevisiae. Curr. Biol. 12: 944-950.

Yoshida, S., Kono, K., Lowery, D.M., Bartolini, S., Yaffe, M.B., Ohya, Y., and Pellman, D. 2006. Polo-like kinase Cdc5 controls the local activation of Rhol to promote cytokinesis. Science 313: 108-111.

Yoshida, S., Bartolini, S., and Pellman, D. 2009. Mechanisms for concentrating Rhol during cytokinesis. Genes \& Dev. 23: 810-823.

Yoshizaki, H., Ohba, Y., Kurokawa, K., Itoh, R.E., Nakamura, T., Mochizuki, N., Nagashima, K., and Matsuda, M. 2003. Activity of Rho-family GTPases during cell division as visualized with FRET-based probes. I. Cell Biol. 162: 223-232.

Yuce, O., Piekny, A., and Glotzer, M. 2005. An ECT2centralspindlin complex regulates the localization and function of RhoA. J. Cell Biol. 170: 571-582.

Zhao, W.M. and Fang, G. 2005. MgcRacGAP controls the assembly of the contractile ring and the initiation of cytokinesis. Proc. Natl. Acad. Sci. 102: 13158-13163. 


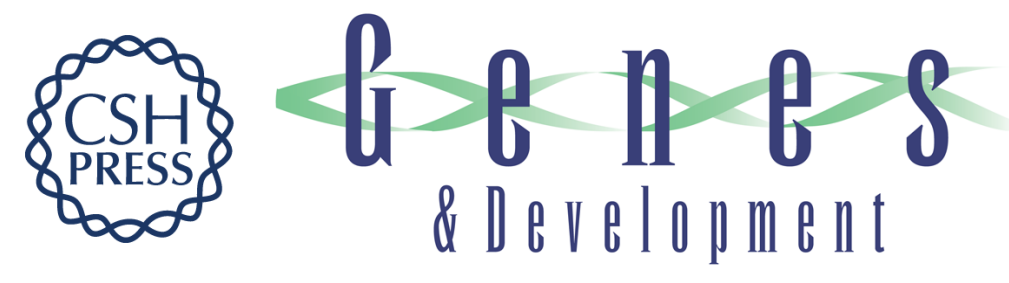

\section{Single cells (put a ring on it)}

Benjamin A. Wolfe and Michael Glotzer

Genes Dev. 2009, 23:

Access the most recent version at doi:10.1101/gad.1801209

References This article cites 39 articles, 17 of which can be accessed free at: http://genesdev.cshlp.org/content/23/8/896.full.html\#ref-list-1

License

Email Alerting Receive free email alerts when new articles cite this article - sign up in the box at the top Service right corner of the article or click here.

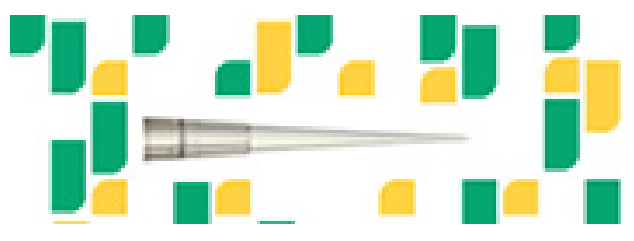

Focused on your science. 